\title{
Treatment of ischemic stroke with r-tPA: implementation challenges in a tertiary hospital in Brazil
}

Tratamento do acidente vascular cerebral isquêmico com r-tPA: o desafio da implantação em um hospital terciário no Brasil

Elza Dias Tostaํ, Letícia Costa Rebello², Soraya Soares Almeida², Márcia Silva Santos Neiva²

\begin{abstract}
Purpose: This paper presents the initial experience with thrombolysis for acute ischaemic stroke at Hospital de Base do Distrito Federal (HBDF), Brazil, and the difficulties associated with the implementation of this treatment. Method: A retrospective study was performed using the medical records of all patients with acute stroke who were treated with intravenous alteplase in our department, between May 2011 and April 2012. Results: The thrombolytic therapy was administered to 32 patients. The mean time between the ictus and the start of stroke therapy start was 195 (60-270) minutes. Sixteen patients demonstrated a significant clinical improvement (decrease in National Institute Health Stroke Scale [NIHSS] score $\geq 4$ points in 24 hours); 6 patients were discharged with an NIHSS score of 0 and 2 demonstrated haemorrhagic transformation. Conclusions: The results of our study are similar to those reported in the literature, although we have been dealing with difficulties, such as the lack of a stroke unit.
\end{abstract}

Keywords: stroke, thrombolytic therapy, tissue plasminogen activator.

RESUMO

Introdução: O objetivo deste trabalho é apresentar a experiência inicial em trombólise para o acidente vascular encefálico isquêmico (AVC) no Hospital de Base do Distrito Federal (HBDF) e as dificuldades encontradas na implementação do serviço. Método: Foi realizado estudo retrospectivo através da análise de prontuário de todos os pacientes submetidos à terapia trombolítica no período de maio de 2011 a abril de 2012. Resultados: A terapia trombolítica foi administrada a 32 pacientes. 0 tempo médio do ictus até o início da terapia foi de 195 (60270) minutos. Dezesseis pacientes apresentaram uma melhora clínica significativa (queda do National Institute Health Stroke Scale [NIHSS] maior ou igual a 4 pontos em 24 horas). Seis pacientes receberam alta com NIHSS 0. Dois pacientes apresentaram transformação hemorrágica. Conclusão: Apesar das dificuldades encontradas, como a ausência de uma Unidade de AVC, o desfecho da nossa série é semelhante aos resultados descritos na literatura.

Palavras-chave: acidente vascular cerebral isquêmico agudo, trombólise intravenosa, rt-PA.

Stroke is one of the greatest public health problems in the world. Medical research has made significant progress in the treatment of acute stroke in the last 10-15 years. Presently, among the possible stroke treatments with a strong level of supportive evidence are the use of thrombolytics and hospitalization in a stroke treatment unit ${ }^{1}$.

The publication of Brazil's Ordinance number 664 (April 13, 2012) established a new care protocol for patients with ischaemic or haemorrhagic stroke. This law incorporated alteplase (r-tPA) in the list of medications provided by the Unified
Health System in Brazil (Sistema Único de Saúde - SUS). This medication is important in stroke treatment, worldwide. Alteplase has been available at the Hospital de Base do Distrito Federal (HBDF) since 2011 and its first use for thrombolysis in a stroke patient occurred in May 2011.

The objective of this paper was to report the experience of the HBDF Neurology Unit with the implementation of intravenous thrombolysis, using r-tPA, between May 2011 and April 2012, and to show the difficulties encountered in this process.

\footnotetext{
1President of the Brazilian Academy of Neurology, Brasilia DF, Brazil;

${ }^{2}$ Serviço de Neurologia, Hospital de Base do Distrito Federal, Brasília DF, Brazil.

Correspondence: Elza Dias Tosta; SQN 111 / Bloco 1 / apto 406; 70754-090 Brasília DF, Brasil; E-mail: marciassantos2005@yahoo.com.br

Conflict of interest: There is no conflict of interest to declare.

Received 21 August 2013; Received in final form 20 December 2013; Accepted 17 January 2014.
} 
Table 1. Summary of patient ages, symptom-to-door, door-to-needle, and symptom-to-needle times.

\begin{tabular}{|c|c|c|c|c|c|c|}
\hline & \multirow{2}{*}{ Mean } & \multirow{2}{*}{ SEM } & \multicolumn{2}{|c|}{$95 \% \mathrm{Cl}$} & \multirow{2}{*}{ Min } & \multirow{2}{*}{ Max } \\
\hline & & & Lower & Upper & & \\
\hline Age & 60.0 & 2.5 & 54.8 & 65.2 & 31 & 86 \\
\hline Symptom-to-door & 118.0 & 10.9 & 95.6 & 140.5 & 11 & 228 \\
\hline Door-to-needle & 89 & 8.9 & 71.6 & 108.3 & 32 & 244 \\
\hline$\Delta$ t (Symptom-to-needle) & 195 & 12.0 & 170.6 & 219.8 & 60 & 270 \\
\hline
\end{tabular}

\section{METHOD}

For this research, a retrospective study was performed to analyse the medical records of all acute stroke patients treated with intravenous r-tPA, in our department, between May 2011 and April 2012. We determined the time interval between the onset of stroke symptoms and the admission to hospital and the start of r-tPA treatment. We analysed each patient's National Institutes of Health stroke scale (NIHSS) score before thrombolytic treatment, 24 hours after the start of therapy and when the patient was discharged. We also evaluated each patient's modified Rankin Scale (mRS) score upon discharge. Our results were compared with data from the literature.

Data were collected from the database of stroke patients receiving r-tPA treatment, using a standard data collection form. In addition, one investigator was responsible for checking all items of interest, thereby minimizing collection bias and ensuring full completion of the form.

\section{Statistical analysis}

Data were tabulated in a Excel $^{\text {B }} 2010$ for Windows (Microsoft, Redmond, WA, USA) worksheet and exported to SPPS $^{\mathbb{E}}$ v20 (SPSS, Chicago, IL, USA). Distribution of the data was evaluated by Kolmogorov-Smirnov tests. Normally distributed data are expressed as means \pm SEM, and non-normally distributed data are expressed as medians (interquartile range).

\section{RESULTS}

In the first year of thrombolysis implementation (between May 2011 and March 2012) 32 patients underwent treatment. Among them, there was a predominance of males (19; 59\%). We evaluated the time intervals between symptom start and hospital arrival (symptom-to-door time), between hospital arrival and the start of infusion (door-to-needle time), and between the start of symptoms and treatment with r-tPA (delta $\mathrm{T}$ or symptom-needle time). The NIHSS was used in the initial evaluation of patients, at 24 hours, and when the patients were discharged. These results are described in Tables 1 and 2 .

The results indicated that 16 patients showed a significant clinical improvement, defined as a decrease in the
NIHSS score of $>4$ points within 24 hours. In addition to the patients showing significant improvement within the first 24 hours, 5 patients showed continued clinical improvement until they were discharged; 6 patients were discharged with a NIHSS score of 0 .

Among the treated patients, 2 (6\%) developed haemorrhagic transformation, associated with thrombolysis, but did not die. Another 3 patients were transferred to the intensive care units of other institutions and were lost to follow-up. Among the 4 patients who died, 2 developed intracranial hypertension due to a malignant stroke, 1 developed fatal intracerebral haemorrhage 8 days after beginning thrombolysis, and 1 patient died due to clinical complications. Therefore, none of the bleeding events was immediately related to the r-tPA treatment.

We used the mRS to functionally evaluate the treated patients at the time of their discharge. The patients with a mRS of 0-2 were considered to have had a favourable outcome, and accounted for $44 \%$ (14 patients) of the patients studied.

The difficulties associated with the implementation of intravenous thrombolysis, in our hospital, are listed in Table 3.

\section{DISCUSSION}

Globally, cerebrovascular disease is the third most common cause of death and the leading cause of long-term disability. Studies have estimated that 795,000 people suffer strokes each year in the United States and that one in every 17 deaths is caused by cerebrovascular disease ${ }^{1}$. In Brazil, in 2005, acute ischaemic strokes accounted for $10 \%$ of all deaths (90,006 deaths) and $10 \%$ of all admissions to public

Table 2. Analysis of patient National Institutes of Health Stroke Scale (NIHSS) scores.

\begin{tabular}{lcccc}
\hline NIHSS & Median & Q1 & Q3 & IQR \\
\hline Before & 15.0 & 6.3 & 18.8 & 13 \\
After & 10.0 & 3.5 & 16.0 & 13 \\
24 hours & 5.5 & 2.0 & 14.3 & 12 \\
48 hours & 2.0 & 0 & 8.5 & 9 \\
Discharge & 2.0 & 0 & 8.0 & 8 \\
\hline
\end{tabular}

Q1: First quartile (25\%); Q3: Third quartile (75\%); IQR: Interquartile Range. 
Table 3. Main difficulties associated with implementing alteplase therapy at Hospital de Base do Distrito Federal, Brazil.

1. Lack of an organized stroke unit

2. Few neurologists trained in thrombolysis

3. Magnetic Resonance lack at Emergency Centre for doubtful cases and for end window

4. Lack of coagulation tests within the Emergency Centre

5. Lack of a common protocol for the management of bleeding complications

6. Lack of a neuroradiologist in the Emergency Centre

7. Lack of a neurological intensive care unit for patients receiving thrombolytic therapy

8. Lack of a hemodynamic team in the Emergency Centre

9. Lack of stroke-related training programs for other health professionals

hospitals ${ }^{2}$, making it the number one cause of death and disability in the country.

Stroke is a major public health problem, but strokerelated research remains infrequent in comparison with that directed towards heart disease and cancer. In spite of this, advances in acute stroke management have occurred over the last 10-15 years. Undoubtedly, an important contribution has been the advent of stroke centres, which are effective for the management of all stroke types and which provide specialized professional care, reducing mortality by $20 \%$ and improving functional outcomes ${ }^{3}$.

A high degree of evidence (level IA) supports the current mainstays of stroke treatment, namely, the use of thrombolytics and the hospitalization of patients in stroke care units $^{3}$. The Stroke Unit Trialists' Collaboration, ${ }^{4}$ in a metaanalysis of 30 randomized trials, showed that patients maintained in stroke units have better chances of survival, are able to return to their homes, and achieve higher levels of independence in activities of daily living. According to the study, mortality was reduced by $18 \%$, dependency decreased by $29 \%$, and the need for patient institutionalization declined by $25 \%$ for patients treated in stroke units compared with those treated in regular wards.

As previously stated, the publication of Brazil's Ordinance $664 / 2012$ established a new care protocol for patients with ischaemic and haemorrhagic stroke. With the publication of this legal measure, r-tPA was incorporated into the list of drugs provided by the Brazilian public health system, an important milestone for the treatment of patients with stroke. However, prior to this ordinance, the Distrito Federal government had approved r-tPA for use by the HBDF Neurology Service, and implementation of therapeutic thrombolysis at the HBDF started in 2011, with the first procedure performed in May.

Intravenous thrombolysis treatment is the first choice for acute ischaemic stroke patients, who arrive at the hospital within 4.5 hours after symptom onset; better results are obtained in patients with small vessel occlusion. In most instances, the induced recanalization by intravenous r-tPA occurs within the first hour after the start of treatment; the chances of recanalization drop significantly after the first 60 minutes. Theoretically, intra-arterial thrombolysis (IA) can provide a higher dose of the thrombolytic agent directly to the clot, with fewer systemic complications and higher rates of recanalization ${ }^{5,6}$, and is being performed in some centres as a rescue treatment for large vessel occlusion. The IA treatment extends the therapeutic window and provides an option for patients with contraindications for systemic thrombolysis treatment and for patients who failed intravenous thrombolysis ${ }^{5,6}$. However, this treatment modality is not available in our service since it is mandatory a scale of doctors on call for interventional treatment 24/7.

Countless difficulties were encountered during the implementation of thrombolytic therapy at HBDF. The HBDF Neurology Unit functions with an attending physician, who is responsible for all emergencies and for the support of the neurology resident; the resident is responsible for attending to the patients that have already been admitted. Within the area, HBDF is the only hospital with emergency neurology care available 24/7. Thus, the volume of neurologic emergencies is very high, overloading the doctor on duty. This was one of the difficulties encountered during the implementation of the Stroke Protocol and early thrombolytic treatment. This issue could be resolved by the recruitment of another neurologist to share the workload. Another challenge was the absence of a common protocol, between the Neurology and Neurosurgery departments, for treating post-thrombolysis complications, such as intraparenchymal haemorrhage.

Table 3 shows other difficulties encountered during the implementation process. One of the difficulties described in previously reported studies is the challenge of reproducing clinical trial results in real-world conditions. When a clinical trial is conducted, a specialist is expected to be available, in conjunction with a multidisciplinary team and motivated staff within the stroke unit; this cannot be easily replicated in daily practice ${ }^{7}$. Another reported issue was the difficulty in standardizing the procedures for acute stroke patients and improving the level of care for these patients ${ }^{8}$.

Despite the difficulties encountered during the implementation process, our results were similar to those published in the literature, when compared with other studies in developing countries. We evaluated studies conducted in Tel Aviv, Israel'; Pathum Thani, Thailand ${ }^{10}$ and Curitiba, Brazil $^{11}$. Among these studies, the mean patient age was similar to that for patients suffering strokes in our study (mean age, 59 years). As expected, all patients were predominantly male. Patients with greater NIHSS scores were identified in the Thai study (NIHSS, 34). Differences in the symptom-todoor time, door-to-needle time, and needle application time, were observed, with our results being longer than those 
Table 4. Comparison of the final outcomes at the Hospital de Base do Distrito Federal (HBDF) and in the CASES/SITS-MOST clinical studies.

\begin{tabular}{lccc} 
& HBDF & SITS-MOST & CASES \\
\hline Patient numbers & 32 & 6483 & 1135 \\
Study period (months) & 12 & NR & 30 \\
Mean Age (years) & 59 & 68 & 73 \\
Variation & $(31-86)$ & $(59-75)$ & $(63-80)$ \\
Gender (\% male) & 59 & 60 & 55 \\
NIHSS variation & $3-24$ & 72 NR & $9-19$ \\
Symptom-door time (min) & $118(11-228)$ & 68 NR & NR \\
Door-to-needle time (min) & $89(32-244)$ & $140(115-165)$ & $85(60-109)$ \\
Symptom-needle time (min) & $195(60-270)$ & $55 \%$ & $155(130-175)$ \\
Significant improvement in NIHSS & $50 \%$ & $39 \%$ & NR \\
Favourable result at discharge (mRS 0-2) & $44 \%$ & $17 \%$ & NR \\
Haemorrhagic transformation & $6 \%$ & $11.30 \%$ & $28.9 \%$ \\
Mortality & $13 \%$ & $3.6 \%$ \\
\hline
\end{tabular}

NR: not reported.

reported in the other studies. These differences highlight the need for educational programs for teaching people to recognize the signs and symptoms of stroke, and the need for better interaction between the emergency services team and the hospital team to optimize the door-to-needle time.

In February 2012, the Brazilian Cerebrovascular Diseases Society published the Guidelines for Ischemic Stroke Treatment - Part I, which recommended times for patient care in the emergency room ${ }^{2}$.These guidelines recommended times between hospital admission and medical evaluation (10 min), hospital admission and end of cranial computed tomography (CT) (25 min), admission and cranial CT interpretation (45 min), admission and r-tPA infusion (60 $\mathrm{min})$, and for the availability of a neurologist (15 $\mathrm{min}$ ) and a neurosurgeon (2 hours). During our thrombolytic therapy implementation period, our door-to-needle time was longer than that recommended by the guidelines, confirming the need for better organization of the service. However, the neurologist and neurosurgeon in our service were on call, resulting in the enhanced availability of these specialists, relative to the times recommended by the guidelines.

Comparing our results with those of larger studies, the data are similar (Table 4). Our average door-to-needle time was similar to that found in the CASES study ${ }^{12}$, while the symptom-to-needle time was much longer than that reported in the CASES and SITS-MOST studies ${ }^{3}$. This finding also indicates the need for educational programs to increase stroke awareness among the general population and to aid in the early recognition of the signs and symptoms of strokes.r-tPA treatment costs were higher than for conservative treatment, largely reflecting the cost of the drug. An important consideration is that this additional cost compensates for the lower rehabilitation costs and lower loss of patient productivity within the first 2 years. In addition, patients treated with r-tPA have fewer sequelae than those receiving conservative treatment. Even so, there is a lack of Brazilian studies that have addressed the cost-effectiveness of thrombolytic treatment. In this regard, a key paper was published, in 2010, by a team from Rio de Janeiro and Porto Alegre. They concluded that thrombolytic treatment with r-tPA within the first 3 hours after a stroke ictus is cost-effective in Brazil. During the first year after a stroke, patients treated with r-tPA had higher overall treatment costs than patients treated conservatively, likely reflecting the cost of the drug. From the second year onward, the direct and indirect costs were lower in patients receiving thrombolytic therapy than in those receiving conservative care ${ }^{13}$.

The implementation of thrombolytic therapy involves multiple levels of care and commitment by an interdisciplinary team. Thus, problems, such as those found in our study, are common during these implementation processes. As mentioned in another study, the availability of a neurologist with expertise in vascular disease on the attending staff of a tertiary care hospital is unlikely. With the implementation of a specific stroke unit ward, this problem is partially solved since it forces compliance with the protocols established by the service, improving both the quality of care and patient outcomes. This will be one of the main challenges for HBDF in the coming years.

Despite the difficulties encountered during the implementation of thrombolytic therapy at HBDF, the analysis of the difficulties and outcomes was productive and showed results similar to those reported in the literature. One of the steps towards improving treatment is the recognition of the difficulties and subsequent efforts to manage them in the best way possible. 


\section{References}

1. Marsh JD, Keyrouz SG. Stroke prevention and treatment. J Am Coll Cardiol 2010;56:683-691.

2. Oliveira-Filho J, Martins SC, Pontes-Neto OM, et al. Guidelines for acute ischemic stroke treatment: part I. Arq Neuropsiquiatr 2012;70:621-629.

3. European Stroke Initiative Executive Committee; EUSI Writing Committee, Olsen TS, et al. European Stroke Initiative Recommendations for Stroke Management-update 2003. Cerebrovasc Dis 2003;16:311-337.

4. Wahlgren N, Ahmed N, Dávalos A, et al. Thrombolysis with alteplase for acute ischaemic stroke in the Safe Implementation of Thrombolysis in Stroke - Monitoring Study (SITS-MOST): an observational study. Lancet 2007;369:275-282.

5. Pi Y, Zhang L, Yang Q, et al. Neurothrombectomy for the treatment of acute ischemic stroke in 1530 patients. J Clin Neurosci 2012;19:1363-1368.

6. Khalessi AA, Natarajan SK, Orion D, et al. Acute stroke intervention. JAAC: Cardiovasc Intervent 2011;4:261-269.

7. Seenan P, Long M, Langhorne P. Stroke units in their natural habitat. systematic review of observational studies. Stroke 2007;38:1886-1892
8. Alberts MJ, Hademenos G, Latchaw RE, et al. Recommendations for the establishment of primary stroke centers. Brain Attack Coalition. JAMA 2000;283:3102-3109.

9. Gur AY, Shopin L, Bornstein NM. Lessons learned from 2 years experience in intravenous thrombolysis for acute ischemic stroke in single tertiary medical center. Isr Med Assoc J 2009;11:714-718.

10. Muengtaweepongsa S, Dharmasaroja P, Kummark U. Outcomes of intravenous thrombolytic therapy for acute ischemic stroke with an integrated acute stroke referral network: initial experience of a community-based hospital in a developing country. J Stroke Cerebrovasc Dis 2012;21:42-46.

11. Lange MC, Zétola VF, Parolin MF, et al. Curitiba acute ischemic stroke protocol: a university hospital and EMS initiative in a large Brazilian city. Arq Neuropsiquiatr 2011;69:441-445.

12. Hill MD, Buchan AM; Canadian Alteplase for Stroke Effectiveness Study (CASES) Investigators. Thrombolysis for acute ischemic stroke: results of the Canadian Alteplase for Stroke Effectiveness Study (CASES). CMAJ 2005;172:1307-1312

13. Araujo DV, Teich VPassos F, Martins SC. Analysis of the cost effectiveness of thrombolysis with alteplase in stroke. Arq Bras Cardiol 2010;95:12-20 